\title{
Investigation of higher spanwise Helmholtz resonance modes in slender covered cavities
}

\author{
A. T. de Jong ${ }^{\text {a) }}$ and H. Bijl \\ Faculty of Aerospace Engineering, Delft University of Technology, P.O. Box 5058, 2600 GB Delft, \\ The Netherlands
}

(Received 26 October 2009; revised 8 July 2010; accepted 9 July 2010)

\begin{abstract}
Cavity aeroacoustic noise is relevant for aerospace and automotive industries and widely investigated since the 1950s. Most investigations so far consider cavities where opening length and width are of similar scale. The present investigation focuses on a less investigated setup, namely cavities that resemble the door gaps of automobiles. These cavities are both slender (width much greater than length or depth) and partially covered. Furthermore they are under influence of a low Mach number flow with a relatively thick boundary layer. Under certain conditions, these gaps can produce tonal noise. The present investigation attempts to reveal the aeroacoustic mechanism of this tonal noise for higher resonance modes. Experiments have been conducted on a simplified geometry, where unsteady internal pressures have been measured at different spanwise locations. With increasing velocity, several resonance modes occur. In order to obtain higher mode shapes, the cavity acoustic response is simulated and compared with experiment. Using the frequency-filtered simulation pressure field, the higher modes shapes are retrieved. The mode shapes can be interpreted as the slender cavity self-organizing into separate Helmholtz resonators that interact with each other. Based on this, an analytical model is derived that shows good agreement with the simulations and experimental results. (C) 2010 Acoustical Society of America. [DOI: 10.1121/1.3473698]
\end{abstract}

PACS number(s): 43.28.Ra, 43.25.Gf, 43.28.Hr [AH]

Pages: 1668-1678

\section{INTRODUCTION}

Cavity geometries under influence of a grazing flow can produce aeroacoustic noise. The mechanism for this noise is dependent on the cavity geometry and flow properties. In literature, most focus has been on certain geometry types (various non-slender cavities) due to their relevance in aerospace and automotive industries.

In the aircraft industry, most focus has been put on open shallow cavities. These cavities resemble aircraft weapon bays and landing gear wheel wells. ${ }^{1}$ An example outside the aircraft industry is the noise from the pantograph recess in trains. $^{2}$ Deep cavity resonance has also been a topic of interest, for example in side branches of pipe systems. ${ }^{3,4}$ Covered cavity geometries have been investigated in detail, where the cavity often behaves like a Helmholtz resonator. For example Dequand et $a l^{5,6}$ investigated the resonance lock-on amplitude of several rectangular Helmholtz resonator geometries under a thin boundary layer flow. Examples of applications are the sound generation in flute like instruments, ${ }^{7-9}$ the buffeting of open car sunroofs and side windows ${ }^{10,11}$ and Helmholtz resonators used in acoustic liners. ${ }^{12}$

The current investigation concerns a special setup relevant in the automobile industry, the tonal noise from automobile gaps. These covered cavities are slender (width much larger than length or depth) and under influence of a low Mach number flow $(M \approx 0.1)$ with a relatively thick boundary layer. Both slenderness and a thick boundary layer reduce the resonance response of a cavity. Howe's theory ${ }^{13}$

\footnotetext{
a) Author to whom correspondence should be addressed. Electronic mail: a.t.dejong@tudelft.nl
}

indicated that an increased aspect ratio modifies the impedance, thereby reducing resonance. Kooijman et al. ${ }^{14}$ showed that by increasing the boundary layer momentum thickness compared to the opening length, the instability of the opening shear layer is reduced. In fact, in general door gaps only show a passive response to the flow, although there are cases where a resonance lock-on can occur.

Door gap structures have not been examined in great detail, although quite some investigations on geometries of this kind can be found in literature. For example Nelson ${ }^{15,16}$ experimentally analyzed a Helmholtz resonator with laser Doppler velocimetry, where an opening slot of 600 by 10 $\mathrm{mm}$ was excited by a grazing flow of 16 to $27 \mathrm{~m} / \mathrm{s}$. In addition, Henderson ${ }^{17,18}$ presented benchmark experimental data of a resonator with a 460 by $8 \mathrm{~mm}$ slot with a thick boundary layer flow of 45 to $60 \mathrm{~m} / \mathrm{s}$. Mongeau et al. ${ }^{19}$ show experimental results of a $25 \mathrm{~cm}$ wide cavity that resembles a door gap, including a seal fixture, where the cavity showed a passive response to the outside flow.

The current investigation is set up to investigate the acoustic resonance behavior over a wide range of velocities in order to reveal a multitude of acoustic resonance modes, with special focus on resonances with spanwise variations that occur. Spanwise modes have been identified in shallow cavities in a large eddy simulation of a weapon bay by Larchevêque, ${ }^{20}$ corresponding to simple spanwise room modes. For covered cavities, Henderson mentioned the possible existence of modes with spanwise variation. But the pressure excitations were measured at a single spanwise location and it was therefore not possible to identify these modes. Mongeau observed passive low amplitude spanwise modes which were not coupled to higher amplitude excita- 
tions. Therefore they do not correspond to spanwise varying resonance modes observed in the current research.

In the present investigation experiments, simulations and analytical studies are combined. First experiments have been conducted on a simplified cavity geometry, where unsteady internal pressures have been measured at different spanwise locations. In order to obtain the experimentally observed higher mode shapes, the cavity acoustic response is simulated and compared with experiment. Using the frequencyfiltered simulation pressure field, the higher modes are identified and an analytical model is derived. Sections III and IV give the experimental flow run setup and results. The numerical acoustic response test is described in Sec. V. The analytical model is derived and compared with the flow run experimental results in Sec. VI.

\section{THEORY: CAVITY RESONANCE MODES}

This section briefly explains the aeroacoustic mechanisms possible in the investigated setup. A cavity volume can act as an acoustic resonator to an excitation source in the cavity neck region.

The excitation can either be due to a feedback mechanism of the perturbed shear layer or due to passive excitation by the pressure fluctuations in the turbulent flow ${ }^{21}$ (turbulent rumble). In case of feedback, the shear layer can roll up into discrete vortices impinging on the opening trailing edge coherently (a Rossiter mode), ${ }^{1}$ or exhibit a flapping shear layer motion. In the low Mach number limit, a feedback mode corresponds to excitation at a fixed Strouhal number $\mathrm{Sr}$ $=f \delta / U_{\infty}$, with $f$ the frequency, $\delta$ the slot opening length and $U_{\infty}$ the free stream velocity. If the excitation frequency is close to a resonance frequency, lock-on can occur and the system can resonate. In case of turbulent rumble the resonance should effectively be independent of velocity.

The resonance method can either be of a Helmholtz type or of a standing wave type. In Helmholtz-like resonance the mass of air in the cavity is coherently compressed and expanded. The equation for a Helmholtz resonator is ${ }^{22}$

$$
f_{H}=\frac{c}{2 \pi} \sqrt{\frac{S}{V L^{\prime}}} .
$$

Here $V$ is the cavity volume, $S$ is the cavity neck surface area and $L^{\prime}$ is the corrected vertical length of the cavity neck. The relation between the real vertical cavity neck height $L$ and $L^{\prime}$ is $L^{\prime}=L+l$, where $l$ is an end correction factor to account for the added resonating mass above and below the opening. For non-slender openings it is based on the surface area $l \propto \sqrt{S}$, whereas in the slotted opening of the current investigation, it is assumed to be related to the opening length only $l \propto \delta$ and independent of the slot width $W{ }^{12}$

Besides Helmholtz-like excitation, where the air in the cavity is coherently pressurized and expanded, standing wave patterns inside the cavity can emerge. The combined effect of all acoustic pressure waves in the cavity volume can create a standing pattern consisting of a fixed number of sinusoidal waves between opposite cavity boundaries. The frequencies for a rectangular box of dimensions $L_{c a v}, D, W$ $\operatorname{are}^{22}$
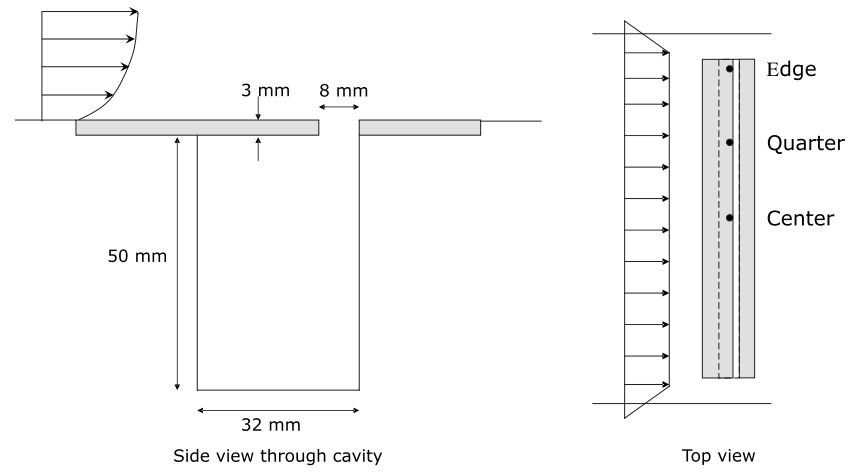

FIG. 1. Cavity dimensions.

$$
f_{n_{x}, n_{y}, n_{z}}=\frac{c}{2} \sqrt{\left(\frac{n_{x}}{L_{c a v}}\right)^{2}+\left(\frac{n_{y}}{2 D}\right)^{2}+\left(\frac{n_{z}}{W}\right)^{2}} .
$$

\section{EXPERIMENTAL SETUP}

The experimental geometry consists of a rectangular cavity partially closed off by a rigid overhang from the leading edge of the cavity. The cavity opening is subjected to a flow with a thick (compared to the opening length $\delta$ ) flat plate boundary layer profile. The aspect ratio of the cavity is large, $W / \delta \gg 1$.

For the experiments the vertical tunnel (V-tunnel), located at Delft University of Technology in the Netherlands was used. The V-tunnel is a tunnel with an open test section and a vertical outflow though a circular opening, $0.6 \mathrm{~m}$ in diameter. Due to the high contraction ratio of the settling chamber the quality of the airflow is high (low turbulence) and the tunnel is relatively silent.

The model used in the windtunnel is a cavity embedded in a splitter plate which has an elliptic nose cone. Figure 1 gives the dimensions of the cavity and Fig. 2 shows a photograph of the splitter plate with the embedded cavity. The expected Helmholtz resonance frequency is around $800 \mathrm{~Hz}$ and expected base resonance onset velocity is $25 \mathrm{~m} / \mathrm{s}$. This is in the velocity range of the used vertical windtunnel ( 0 to 47 $\mathrm{m} / \mathrm{s}$ ) and is also an interesting velocity to resemble a car door gap (typical car highway speeds).

The boundary layer develops on the first section of the plate. By adjusting the length of the splitter plate and the location of trip wires the boundary layer is controlled in a precise and reproducible way. In the setup used for this publication, the splitter plate upstream flat plate section is set to $0.7 \mathrm{~m}$ and a $1 \mathrm{~mm}$ high zigzag type turbulator strip is located $10 \mathrm{~cm}$ from the splitter plate nose.

The cavity itself is constructed out of thick-walled aluminum to ensure enough rigidity to prevent fluid-structure resonance effects. Interchangeable parts are used to alter the neck geometry. The cavity neck is equipped with sharp edges and a leading edge overhang, as depicted in Fig. 1.

The maximum spanwise width of the cavity is set to 0.5 $\mathrm{m}$, which is smaller than the $0.6 \mathrm{~m}$ width of the splitter plate itself. This leaves $5 \mathrm{~cm}$ on both sides (shown in Figs. 1 and 2) to ensure that the end effects of the outlet (the shear layer of the outlet jet) will not reach the cavity region. In this way 


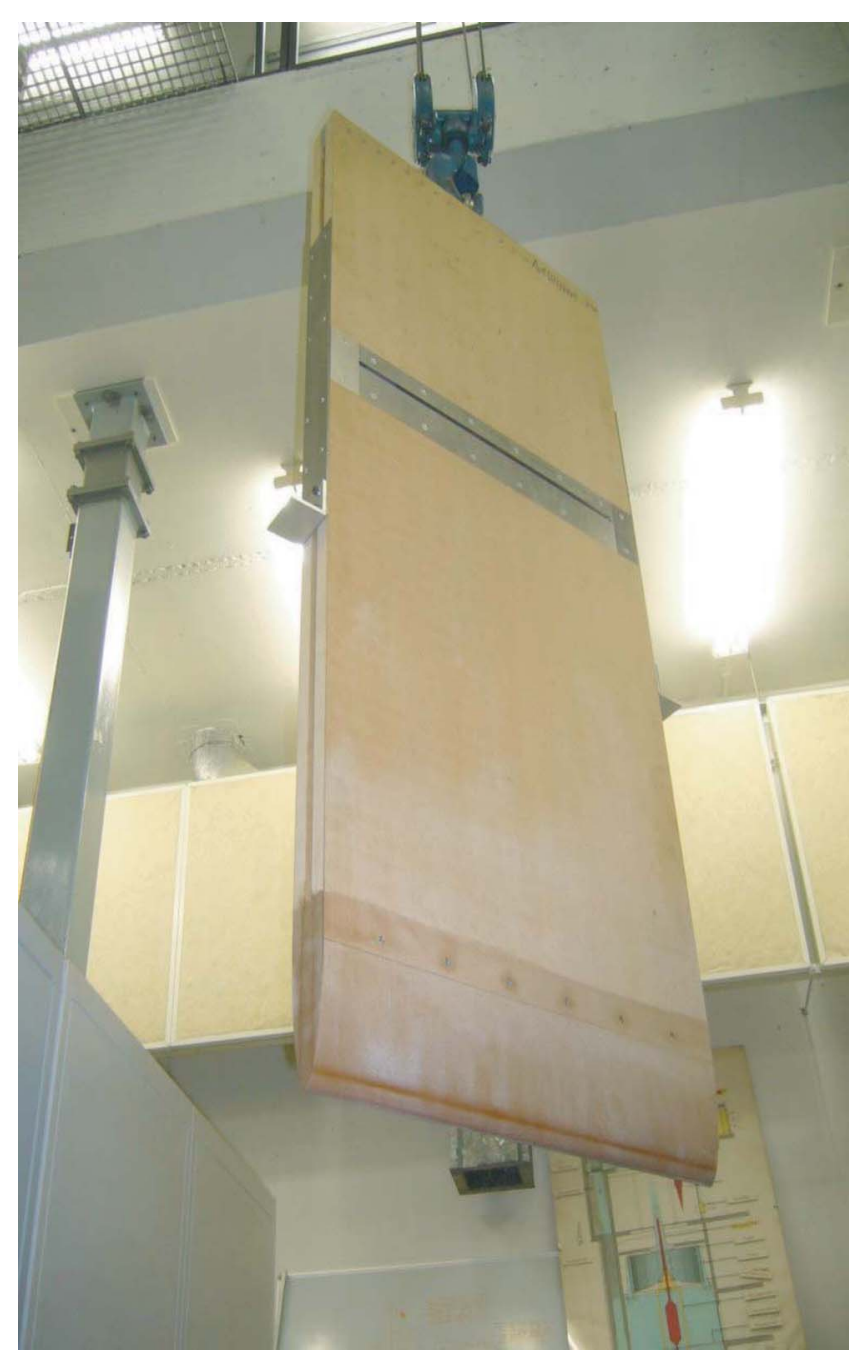

FIG. 2. (Color online) The windtunnel model.

the flow speed and boundary layer shape remain constant along the whole span of the cavity. The current experiments also include runs with a reduced cavity width of $0.3 \mathrm{~m}$ by closing one side with blocks in both the interior and opening. This reduced setup is used to evaluate the effect of the total span on the resonance behavior.
First the cavity has been closed off to determine the boundary layer characteristics. These are measured with a constant temperature hotwire probe. The velocity magnitude in the boundary layer is measured at 25 different heights (with uneven spacing, most measurements in the lower regions), for 4 different flow speeds $(20,24,30$ and $40 \mathrm{~m} / \mathrm{s}$ ) and 3 spanwise locations (center, quarter and edge of cavity, see Fig. 1).

Flow runs with open cavity have been performed to measure the flow induced sound pressure levels inside the cavity. The velocity is increased incrementally, up to the wind tunnel limit of approximately $47 \mathrm{~m} / \mathrm{s}$. The open cavity flow run experiments use 3 pressure transducers. These are located at different spanwise locations (center, quarter, and edge) on the floor of the cavity, as indicated in Fig. 1.

\section{EXPERIMENTAL RESULTS}

In this section the obtained experimental results of the boundary layer properties and cavity internal pressure fluctuations are presented. The cavity measurement data are split into the full span $(0.5 \mathrm{~m})$ and reduced span $(0.3 \mathrm{~m})$ results.

\section{A. Boundary layer profiles}

The boundary layer measurement results are used to check the boundary layer height, shape and fluctuations. This will allow reproducibility of setup conditions for future experiments and simulations. Also, the measurements are used to check that the flow properties are constant across the entire span.

The profiles for the mean and root mean squared (RMS) fluctuation profiles of velocity magnitude (more accurately the vector addition of the streamwise and vertical velocity component) are given in Fig. 3 for the center location (middle of the cavity neck opening, $25 \mathrm{~cm}$ from the opening edge). The other two locations, quarter $(12.5 \mathrm{~cm}$ from the opening edge) and edge have similar boundary layer properties. The figures show that the boundary layer properties re-
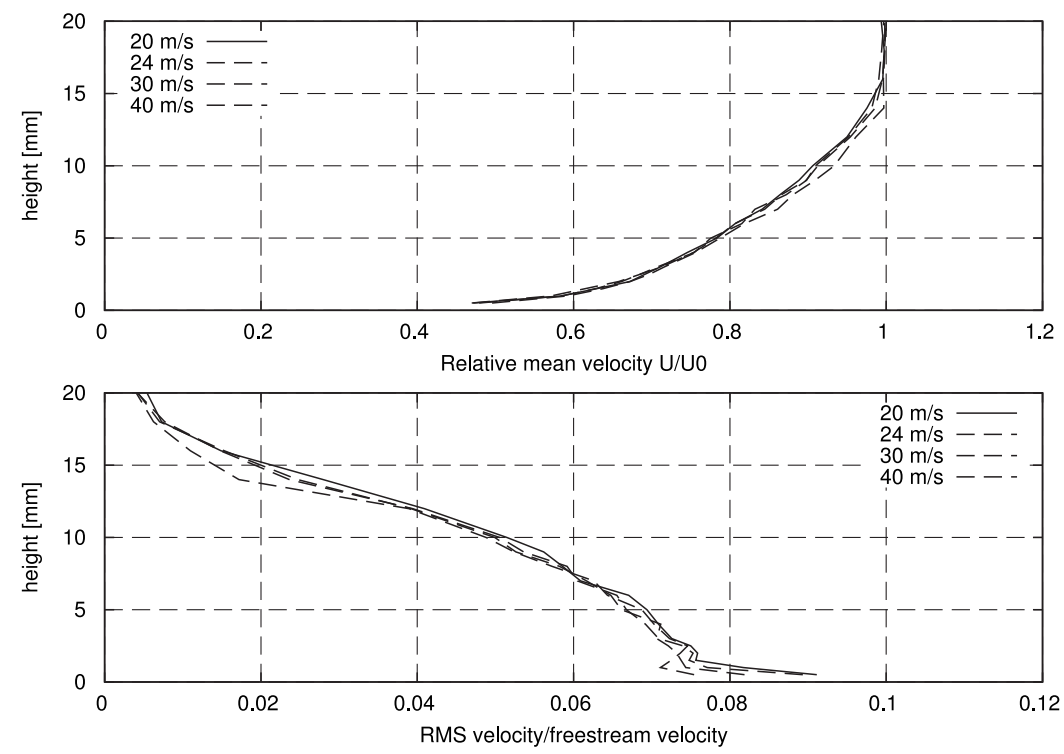

FIG. 3. Experimental boundary layer mean (top) and rms (bottom) profiles of velocity magnitude. 
TABLE I. Overview of boundary layer properties, center/quarter/edge location in span as indicated in Fig. 1.

\begin{tabular}{cccccc}
\hline \hline $\begin{array}{c}\text { Flow velocity } \\
(\mathrm{m} / \mathrm{s})\end{array}$ & Location & $\delta^{*}$ & $\theta$ & $\mathrm{H}$ & $\begin{array}{c}\delta_{99} \\
(\mathrm{~mm})\end{array}$ \\
\hline 20 & Center & 2.46 & 1.80 & 1.36 & 15.4 \\
24 & Center & 2.40 & 1.75 & 1.37 & 14.9 \\
24 & Quarter & 2.50 & 1.80 & 1.39 & 15.2 \\
24 & Edge & 2.43 & 1.78 & 1.37 & 14.9 \\
30 & Center & 2.46 & 1.79 & 1.38 & 15.9 \\
40 & Center & 2.24 & 1.64 & 1.37 & 13.6 \\
\hline \hline
\end{tabular}

main similar during the flow sweep. There is a mild thickness decrease with increasing velocity due to Reynolds effects. $^{23}$

The following integral properties are displayed in Table I: the displacement thickness $\delta^{*}$, the momentum thickness $\theta$, the shape factor $H$ and the height at $99 \%$ of the mean flow $\delta_{99}{ }^{23}$ The displacement thickness $\delta^{*}$, momentum thickness $\theta$, and shape factor $H$ are defined as:

$$
\begin{aligned}
& \delta^{*}=\int_{0}^{\infty}\left(1-\frac{\bar{u}}{U_{e}}\right) d y, \\
& \theta=\int_{0}^{\infty} \frac{\bar{u}}{U_{e}}\left(1-\frac{\bar{u}}{U_{e}}\right) d y, \\
& H=\frac{\delta^{*}}{\theta},
\end{aligned}
$$

where $U_{e}$ is the velocity outside the boundary layer and $\bar{u}$ the local mean velocity magnitude at height $y$. The table includes boundary layer data for 3 different spanwise locations to indicate the consistency across the span.

\section{B. Full cavity span flow runs, $0.5 \mathrm{~m}$ width}

Now that the boundary layer properties are known, the cavity flow induced resonance can be investigated. The cavity resonance is measured for 2 span widths, $0.5 \mathrm{~m}$ and 0.3 m.

This section presents the full $0.5 \mathrm{~m}$ span results and the next section the $0.3 \mathrm{~m}$ reduced span ones.

The flow velocity is increased incrementally. The internal probe sound pressure levels of these velocity sweeps are gathered in spectrograms and given in Fig. 4 for all 3 probe locations. The frequency of the excitation is shown at the vertical axis and the free stream velocity on the horizontal one. The amplitude of the excitation in $\mathrm{dB}$ is indicated by level. The figure shows several resonating modes with increasing velocity and increasing frequency. The first resonance is visible at all probe locations whereas for the higher modes some are not. This indicates a spanwise variation in the higher resonance modes.

From Fig. 4 it is found that all the center points of the excitation modes show a linear relation between frequency and velocity. The Strouhal number $S r=f L / U_{\infty}$ corresponding to this is approximately 0.3 , indicating that all modes are excited by the first stage hy-drodynamic mode. ${ }^{1}$ No excita- tions of the second stage hydrodynamic mode $(S r \approx 0.7)$ are present, although low amplitude onsets of resonance for this Strouhal number can be observed in the upper left part of the figures as light horizontal lines.

Figure 5 shows the pressure time series of the three cavity probes at four different resonance modes. Some resonances do not show excitations of some of the probes, indicating the presence of pressure nodes at that location. Also for the higher modes the excitations of the probes can be of opposite phase.

In Fig. 5 it can be seen that in the base resonance all probes are in phase with each other and have similar excitation amplitudes. This observation enables us to identify the base mode as a Helmholtz resonance. Using Eq. (1) and the experimental excitation frequency of around 800, the resonator added length is found to be $l=1.8 \delta$.

Even though the acoustic pressure amplitudes can be around $120 \mathrm{~dB}$, it can be calculated that the energy transfer from flow to acoustics is low. ${ }^{5}$ In Fig. 5 the maximum acoustic pressure amplitude is $|p|=30 \mathrm{~Pa}$ and $|p|=100 \mathrm{~Pa}$ for the 22 and $42 \mathrm{~m} / \mathrm{s}$ case respectively. The estimated acoustic velocity amplitude $\left|u_{a c}\right|$ in the neck region for a lumped mass system can be estimated by:

$$
\begin{aligned}
\frac{\left|u_{a c}\right|}{U_{0}} & \approx \frac{1}{U_{0}} \frac{V}{\rho_{0} S}\left|\frac{d \rho}{d t}\right|=\frac{1}{U_{0}} \frac{V}{\rho_{0} S c^{2}}\left|\frac{d p}{d t}\right| \\
& =\frac{1}{U_{0}} \frac{L_{c a v}}{\delta} \frac{2 \pi f D}{c} \frac{|p|}{\rho_{0} c},
\end{aligned}
$$

with $S=\delta W$ the area of the neck opening and $V=L_{\text {cav }} D W$ the cavity volume. Here we used $d p=c^{2} d \rho$ with $c$ the speed of sound.

Substituting $f=800 \mathrm{~Hz}, U_{0}=22 \mathrm{~m} / \mathrm{s},|p|=30 \mathrm{~Pa}$ will give $\left|u_{a c}\right| / U_{0} \approx 1 \cdot 10^{-2}$ and substituting $f=1600 \mathrm{~Hz}, U_{0}$ $=42 \mathrm{~m} / \mathrm{s},|p|=100 \mathrm{~Pa}$ will give $\left|u_{a c}\right| / U_{0} \approx 3 \cdot 10^{-2}$. The low acoustical amplitude makes the lock-on hard to predict. ${ }^{24}$

The higher modes are not simple pure standing waves according to Eq. (2), because standing waves that match the observed spanwise pressure variations do not match the observed frequencies. This is indicated in Table II, where frequencies of standing wave modes that match the phases and node locations indicated in Figs. 4 and 5 are compared to the experimentally observed frequencies. $\lambda$ is the standing mode wavelength. Note that in this table the coherently excited base mode does not match any standing wave mode shape. The exact mechanism for the higher modes is not known yet based on the current experimental information. This is why in the next section the effect of a smaller span is investigated to reveal the influence of the total spanwise width.

\section{Effect of a smaller span on the flow induced response}

In order to investigate the higher modes with spanwise variation in more detail, flow runs with a reduced span of 0.3 $\mathrm{m}$ are conducted. All other dimensions remained the same compared to the $0.5 \mathrm{~m}$ span experiments. The spectrograms of the flow induced response for the edge probe is given in Fig. 6. The modes are all excited by the first stage Rossiter 

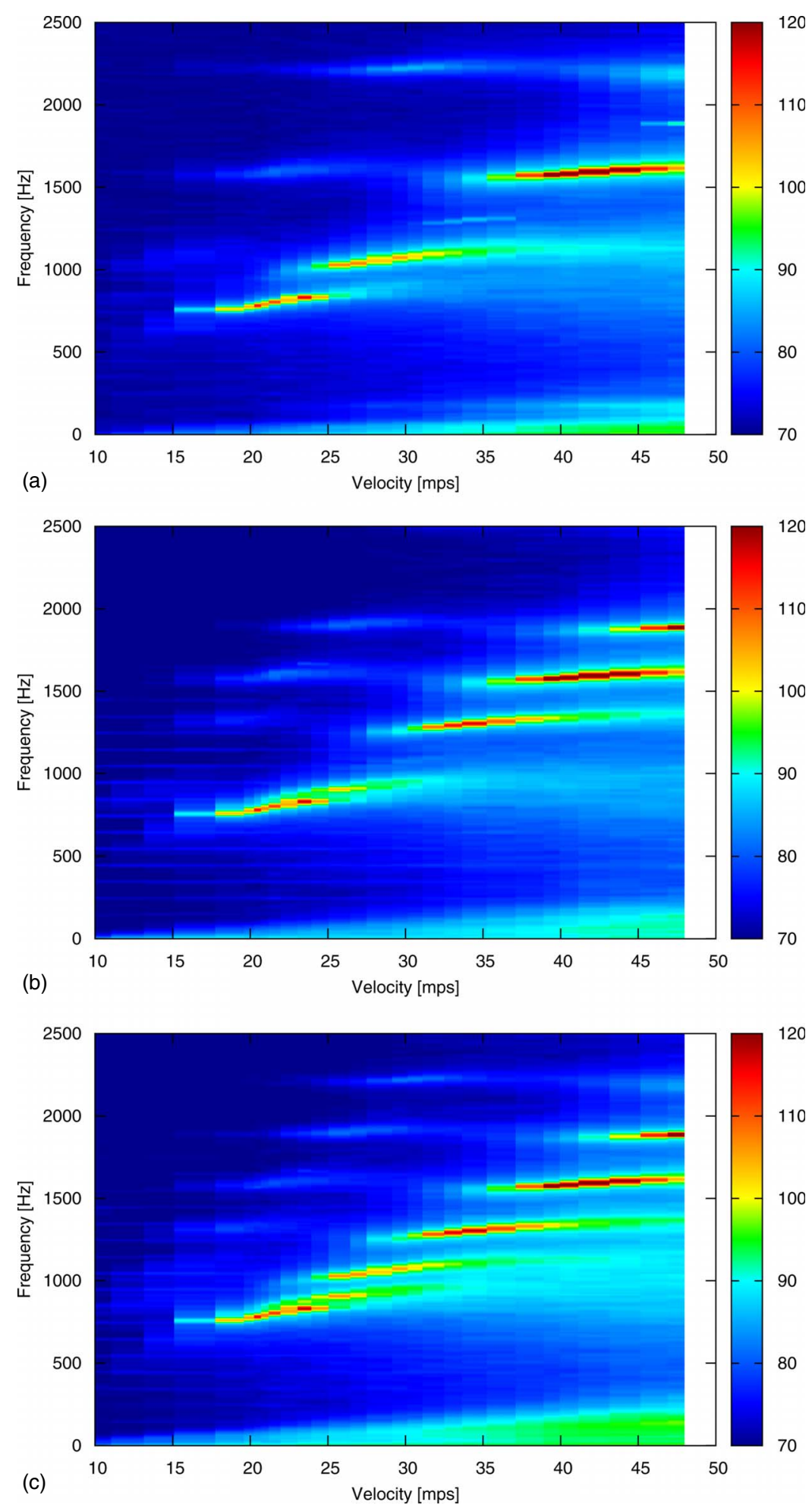

FIG. 4. (Color online) Spectrograms of the three internal pressure transducers, full $0.5 \mathrm{~m}$ span runs, level by sound pressure $(\mathrm{dB})$. mode. The most noticeable differences compared to the 0.5 $\mathrm{m}$ span runs of the lower graph of Fig. 4 are the lower base mode resonance amplitude and location of the higher modes in the diagram.

The base mode has a frequency of around $800 \mathrm{~Hz}$, which is the same as for the $0.5 \mathrm{~m}$ span cavity. This confirms that the slender Helmholtz added length $l$ of Eq. (1) is independent of the cavity width $W$. By comparing the higher modes in the lower graph of Fig. 4 with Fig. 6, a distinct influence of the spanwise width can be observed. The higher modes are shifted to larger velocities and have higher frequencies. For example the second mode shifted from $26 \mathrm{~m} / \mathrm{s}, 900 \mathrm{~Hz}$ to $28 \mathrm{~m} / \mathrm{s}, 1000 \mathrm{~Hz}$ and the third mode shifted from $28 \mathrm{~m} / \mathrm{s}$, $1100 \mathrm{~Hz}$ to $34 \mathrm{~m} / \mathrm{s}, 1400 \mathrm{~Hz}$. The shifts cause less modes to appear in the used velocity interval of $10 \mathrm{~m} / \mathrm{s}$ to $47 \mathrm{~m} / \mathrm{s}$. The smaller span runs reveal strong dependence of the higher mode excitation frequency on the spanwise length. The next section describes an experimental and numerical acoustic response test that will be used to obtain the exact mode shape of the observed modes. 



FIG. 5. Pressure time series of the three cavity internal probes for some of the observed modes.

\section{ACOUSTIC RESPONSE TEST}

This section describes the numerical and the experimental acoustic response test (ART). An acoustic response test provides the acoustic reaction of the cavity setup in the absence of flow. The numerical simulation is used to obtain the exact mode shapes of the cavity resonances. The experimental ART is used to validate the numerical results.

TABLE II. Comparison flow run mode frequencies with pure standing wave modes.

\begin{tabular}{cccc}
\hline \hline Mode & $\begin{array}{c}\text { Exp. freq. } \\
(\mathrm{Hz})\end{array}$ & $\begin{array}{c}\text { Shape matching } \\
\text { st. wave number } \\
n_{s t}=2 W / \lambda\end{array}$ & $\begin{array}{c}\text { St. wave freq. } \\
(\mathrm{Hz})\end{array}$ \\
\hline 1 & 830 & $\mathrm{n} / \mathrm{a}$ & $\mathrm{n} / \mathrm{a}$ \\
2 & 900 & 1 & 340 \\
3 & 1050 & 2 & 680 \\
4 & 1300 & 3 & 1020 \\
5 & 1600 & 4 & 1360 \\
6 & 1900 & 5 & 1700 \\
\hline \hline
\end{tabular}

\section{A. ART setup}

In an ART external speakers emitting a white noise signal are used to excite the cavity in the absence of flow. Microphones inside and outside gather the sound pressure level data.

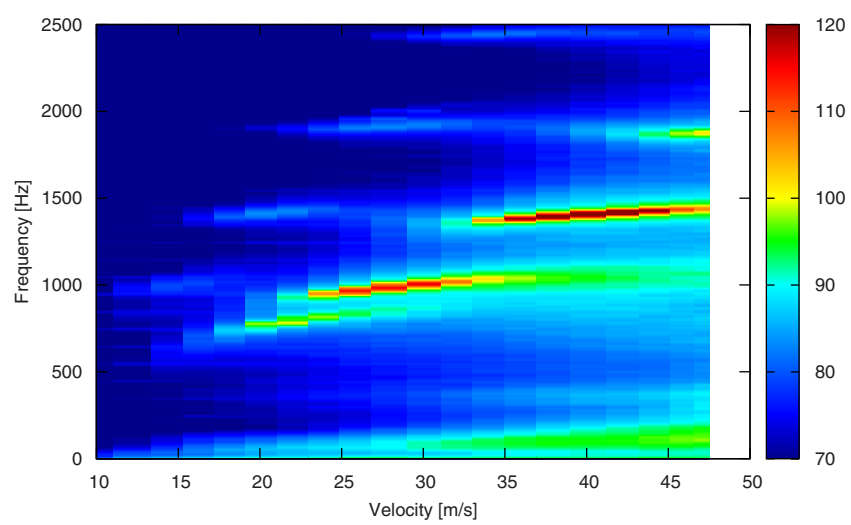

FIG. 6. (Color online) Spectogram of flow run with a $0.3 \mathrm{~m}$ span, edge location internal cavity probe, level by sound pressure $(\mathrm{dB})$. 
The excitation of the cavity can be quantified using the complex transfer function. This is the complex ratio of the sound pressure spectrum inside the cavity to a reference outside the cavity. The resulting complex function in frequency is divided by the complex transfer function of a closed cavity where both the main and reference probe are outside. This last extra step provides a frequency dependent scaling to compensate for transfer losses of sound from the speaker toward the cavity region.

Two important properties of Helmholtz resonators are the resonance frequency $f_{H}$ and the quality factor, $Q$. The resonance frequency is the frequency at which the strongest resonance might occur. The Q-factor describes the sharpness of the resonance peak (as a function of frequency) and can be related to the acoustic impedance of the resonator. The quality factor is defined as:

$$
Q=\frac{f_{H}}{f_{2}-f_{1}},
$$

with $f_{1}$ and $f_{2}$ frequencies at half the amplitude of the resonance frequency, where $f_{1}<f_{H}$ and $f_{2}>f_{H}$.

The experimental and numerical complex transfer ratios are compared with an analytical model for a resonator in order to obtain the resonance frequency $f_{H}$ and quality factor $Q$. The analytical model is fitted with the results using a L2 vector fit in both phase and magnitude. The analytical expression for the transfer magnitude $\|H(f)\|$ is ${ }^{10}$

$$
\|H(f)\|=\frac{1}{k \sqrt{\left(1-\left(f / f_{H}\right)^{2}\right)^{2}+4 D^{2}\left(f / f_{H}\right)^{2}}}
$$

with $k$ the system stiffness and $D$ the damping. The quality factor is related to the damping by $Q=1 / 2 D$.

\section{B. Numerical scheme; The Lattice Boltzmann Method}

The numerical ART is obtained using the Lattice Boltzmann Method (LBM). ${ }^{25}$ LBM is an alternative numerical method to traditional CFD for simulating complex fluid flows. Unlike conventional methods based on macroscopic continuum equations, the LBM starts from mesoscopic kinetic equations, i.e., the Boltzmann equation, to determine macroscopic fluid dynamics. The commercial LBM based package PowerFLOW is used.

The Lattice Boltzmann equation has the following form:

$$
f_{i}\left(\vec{x}+\vec{c}_{i} \Delta t, t+\Delta t\right)-f_{i}(\vec{x}, t)=C_{i}(\vec{x}, t),
$$

where $f_{i}$ is the particle distribution function moving in the $i$ th direction, according to a finite set of the discrete velocity vectors $\left\{\vec{c}_{i}: i=0, \ldots, N\right\} . \vec{c}_{i} \Delta t$ and $\Delta t$ are space and time increments respectively. In the low frequency and longwavelength limit, for a suitable choice of the set of discrete velocity vectors, one can recover the compressible NavierStokes equations through the Chapman-Enskog expansion for Mach numbers less than (approximately) 0.4. ${ }^{25}$ By recovering the compressible Navier-Stokes equations, including an ideal gas equation of state, LBM also inherently recovers acoustics. Some fundamental aeroa-coustic capabilities of the scheme have been studied before, such as wave propagation and compressible behavior. ${ }^{26,27}$ In these cases the code

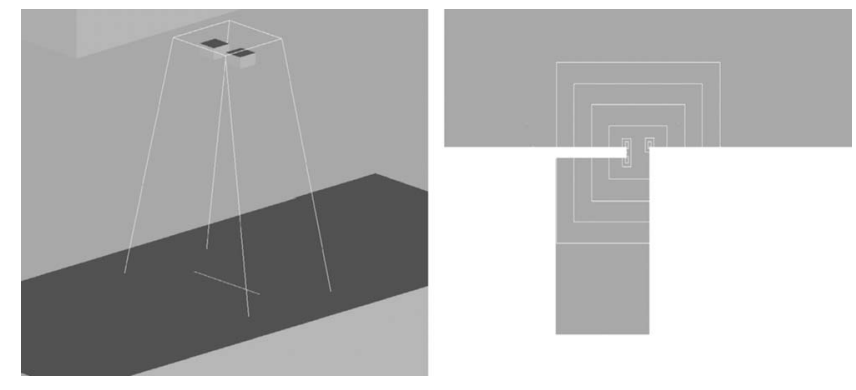

FIG. 7. Acoustic response test simulation setup. The resolution region boundaries are indicated by white contours. Across these boundaries the linear resolution is increased by a factor of 2 , with the finest region having 8 cells $/ \mathrm{mm}$. Two speakers, each with their own white noise signal are suspended $2 \mathrm{~m}$ above the cavity. Left figure gives $3 \mathrm{~d}$ view of domain with speakers in top of figure and cavity slot below, right figure shows a vertical slice through cavity.

has proven itself capable of correctly simulating these acoustics related problems. Examples of the use of the Lattice Boltzmann scheme in acoustics are simulation of radiation from waveguides, ${ }^{28}$ acoustic pulses in flows and duct aeroacoustics, ${ }^{29}$ and side branches. ${ }^{27}$

It should be noted that in the current paper the LBM method is only used for an acoustic response without mean flow, whereas full non-linear flow simulations are possible. Other options to simulate the ART are also possible, such as the boundary element method or finite element method. The authors are planning to perform flow simulations as well in a separate investigation in the future, and therefore LBM is chosen as method of preference.

\section{Numerical setup}

Both the simulated and experimental ART has the same setup and two-step approach of Sec. V A. The full cavity 3d model is included in the simulation and there is no flow present. Two speakers, each with their own independent white noise signal, are used in the numerical setup. Both are positioned at a distance of $2 \mathrm{~m}$ from the cavity (same as in experiment) and are relatively close to each other $(30 \mathrm{~cm}$ between speaker centers). Both the experimental and numerical setup included 2 speakers to ensure excitation of the modes with spanwise variation.

The LBM is solved on a grid composed of cubic volumetric elements, and variable resolution is allowed, where the grid size changes by a factor of two for adjacent resolution regions. Figure 7 gives the resolution regions around the neck of the cavity and speakers. The finest region is located at the sharp edges of the cavity neck and is 8 cells/mm in resolution. This resolution setting has been chosen based on mesh convergence tests on a similar geometry performed by the authors. In addition, the experimental ART is used to validate the simulation setup. In both the experimental and numerical ART the cavity probes are located in the center on the cavity floor.

\section{Comparison ART experiment and simulation}

This section presents the ART results in order to validate the numerical simulation using the experimental ART. Figure 



FIG. 8. Acoustic response test experiment and simulation results of the complex transfer function ratio magnitude (left) and phase (right).

8 shows the phases and magnitudes of the experimental and numerical ART. For both the experimental and numerical ART, the microphones are positioned at the quarter location in span, as indicated in Fig. 1. Table III shows the resonance frequency and quality factor of the complex transfer ratio fits described in Sec. V A. Due to the probe quarter location in the cavity some higher modes cannot be captured in this figure. The sound pressure level of the remaining higher mode around $1600 \mathrm{~Hz}$ is significantly lower and therefore not clearly distinguishable. Both in experiment and simulation there is a second dominant mode at around $3800 \mathrm{~Hz}$ that is not indicated in the figure because it is out of the range of interest.

According to Table III, the base resonance frequency is within 5\% of the experimental results. The acoustic noncompactness of the aperture causes high radiation losses, which accounts for the low observed Quality factors compared to compact aperture resonators. ${ }^{24}$ The similarity between the experimental and numerical base resonance frequency and quality factor validate the use of an acoustic response test simulation to obtain the acoustic properties of slender cavities.

\section{E. ART simulation band-filtered mappings}

The previous section showed that the LBM simulations can predict the acoustic response of the investigated simplified slender cavities. The acoustic simulation results are now used to investigate the observed behavior in experimental flow runs by band-filtering the pressure signal of the ART simulation. In this way maps of acoustic pressure intensity are obtained to check for the acoustic cavity behavior around the higher resonant modes.

Figure 9 shows the result of band-filtering the cavity pressure spectra around the most prominent modes observed in the experimental flow runs. This is displayed in a vertical plane through the resonator center. The used bandwidth is 25 Hz. The pressure maps correspond well to the pressure nodes

TABLE III. Experimental and simulation resonance frequency and quality factor.

\begin{tabular}{lcc}
\hline \hline & $f_{H}$ & \\
& $(\mathrm{~Hz})$ & Q-factor \\
\hline Simulation, sharp edges & 803 & 5.0 \\
Experiment, sharp edges & 828 & 5.9 \\
\hline \hline
\end{tabular}

and phase of the flow experiment probes in Fig. 5. The shape of the modes can be viewed as the cavity subdividing itself into regions acting as separate Helmholtz resonators that interact with each other. The locations corresponding to the end masses of the Helmholtz resonators are then indicated in the figures by the low sound pressure regions and the locations of the Helmholtz resonator volumes themselves are indicated by the high sound pressure regions. In the next section an analytical model of the resonance frequency based on coupled Helmholtz resonators is derived based on these observations.

As mentioned in Sec. V D, the ART complex transfer ratio shows a secondary dominant excitation at around 3800 $\mathrm{Hz}$, an excitation which is not observed in the flow experiments. The band-filtered pressure map of Fig. 10 shows the mechanism for this secondary peak. Instead of a Helmholtz mode, this is a vertical cavity standing wave mode.

\section{ANALYTICAL MODEL OF MULTIPLE HELMHOLTZ RESONATORS}

A lumped mass analytical model of the higher resonance modes is derived using the hypothesis that spanwise sections act as separate coupled Helmholtz resonators. In this section the resonance frequency of a system of $n$ spanwise resonators is determined and compared with the experimentally observed acoustic modes and simulation ART results.

\section{A. Model derivation}

The derivation of the lumped mass analytical model for the multiple Helmholtz resonator mode frequency is related to the derivation of the normal Helmholtz resonator frequency model. It is based on mass conservation in the resonator bodies and momentum conservation in the cavity openings and inter-resonator sections. The equations of a coupled system are explained in this section by using a setup of two spanwise resonators, see Fig. 11. In this figure $m, S$ indicate the masses and surface areas of the moving air columns, $V$ the volumes of the resonator sections and $W$ the widths of the inter-resonator and resonator sections. It is assumed that inter-resonator coupling occurs only within the cavity; acoustic coupling outside the cavity is neglected. Coherent compression is assumed within each resonator body.

The integral mass conservation law applied to one resonator volume can be written as: 


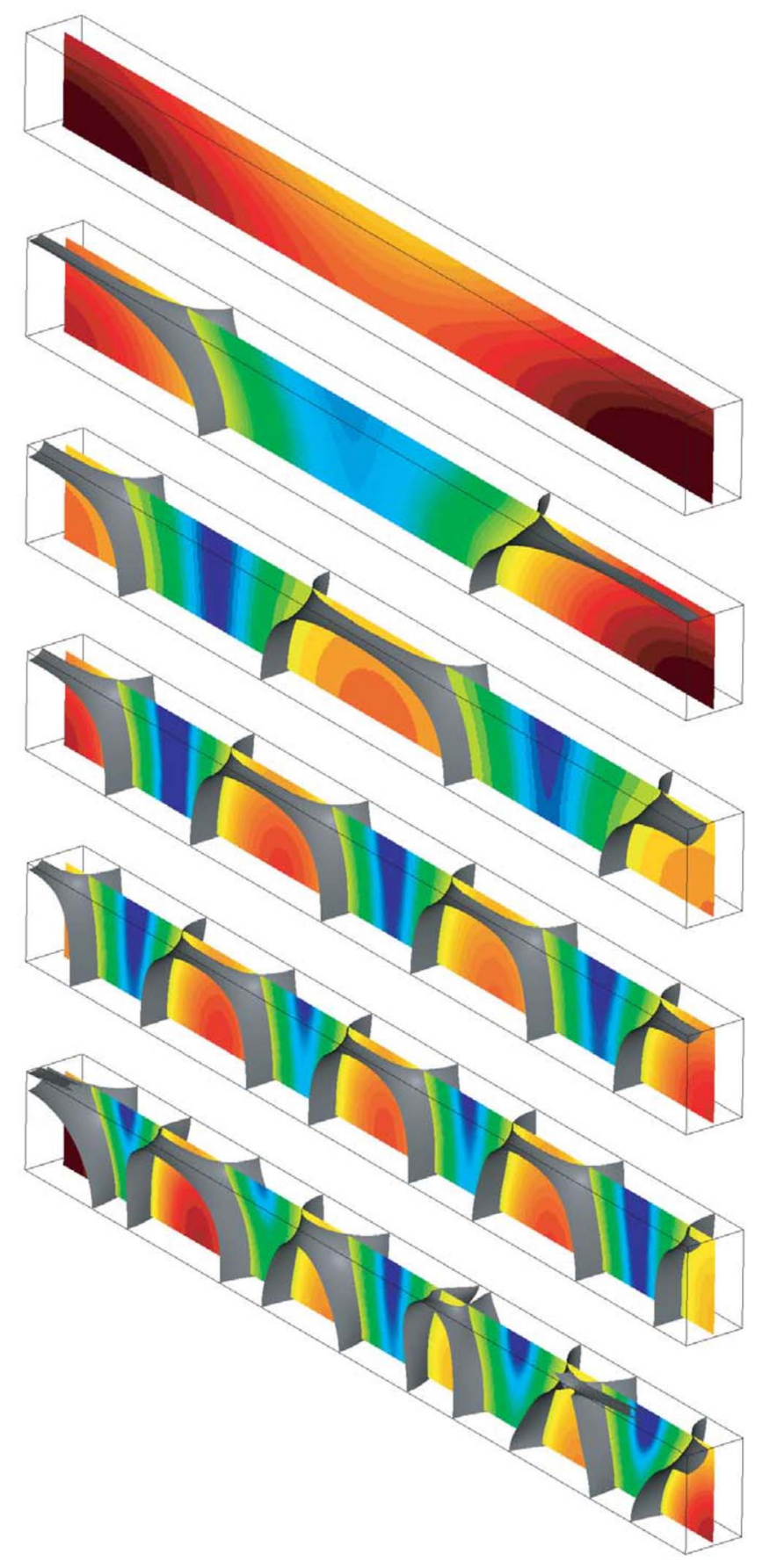

FIG. 9. (Color online) ART simulation band-filtered pressure signals in the cavity on a vertical plane through the cavity neck opening in $\mathrm{dB}$ for 825 , $925,1050,1325,1575$, and $1925 \mathrm{~Hz}$, respectively. Level bandwidth is 20 $\mathrm{dB}$, frequency bandwidth $25 \mathrm{~Hz}$. 3d isosurfaces of constant sound pressure level are included. The cavity opening slot is located on top toward the viewer.

$$
\frac{d}{d t} \iiint_{V} \rho d V=-\iint_{S} \rho \vec{u} \cdot \vec{n} d S
$$

where $S$ is a control surface enclosing $V$ and $\vec{n}$ is the outer normal of $S$. Using a linear approximation and assuming uniform density $\rho$ and velocity $u$ and harmonic disturbances, Eq. (10) can be rewritten for the left and right resonator, respectively, as:
FIG. 10. (Color online) ART simulation band-filtered pressure signals in the cavity on a vertical plane through the cavity neck opening in $\mathrm{dB}$ for the $3800 \mathrm{~Hz}$ excitation standing wave mode. Level bandwidth is $20 \mathrm{~dB}$, frequency bandwidth $100 \mathrm{~Hz}$.

$$
\begin{gathered}
i \omega \frac{V_{1}}{c^{2}} p_{1}=-\rho_{0} u_{1} S_{1}-\rho_{0} u_{3} S_{3}, \\
i \omega \frac{V_{2}}{c^{2}} p_{2}=-\rho_{0} u_{2} S_{2}+\rho_{0} u_{3} S_{3} .
\end{gathered}
$$

The only difference between a completely separate resonator and the coupled system is the right hand term $\rho_{0} u_{3} S_{3}$.

Application of the momentum law in a resonator neck opening yields in linear approximation:

$$
\rho_{0} \frac{\partial u}{\partial t}=-\frac{\partial p}{\partial x} .
$$

Assuming harmonic disturbances will give the following equations for the left and right resonator neck opening:

$$
\begin{aligned}
& i \omega \rho_{0} u_{1}=\frac{p_{1}}{L_{1}^{\prime}}, \\
& i \omega \rho_{0} u_{2}=\frac{p_{2}}{L_{2}^{\prime}} .
\end{aligned}
$$

Here $L^{\prime}=L+l$ is the modified height of the oscillation mass in the cavity neck, as also used in Eq. (1). Application of the momentum law on the coupled mass in the center section yields:

$$
i \omega \rho_{0} u_{3}=\frac{p_{1}-p_{2}}{W_{3}}
$$

The two mass conservation Eqs. (11) and (12) and the three momentum conservations Eqs. (14)-(16) yield the following system:

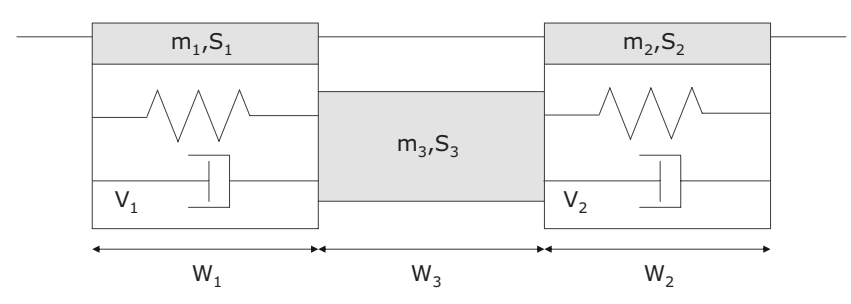

FIG. 11. Two coupled spanwise Helmholtz resonators.

de Jong and Bijl: Higher resonance modes in slender covered cavities 


$$
\begin{aligned}
& {\left[\begin{array}{lllll}
i \omega \frac{V_{1}}{c^{2}} & \rho_{0} S_{1} & 0 & 0 & \rho_{0} S_{3} \\
1 & -i \omega \rho_{0} L_{1}^{\prime} & 0 & 0 & 0 \\
0 & 0 & i \omega \frac{V_{2}}{c^{2}} & \rho_{0} S_{2} & -\rho_{0} S_{3} \\
0 & 0 & 1 & -i \omega \rho_{0} L_{2}^{\prime} & 0 \\
1 & 0 & -1 & 0 & i \omega \rho_{0} W_{3}
\end{array}\right]\left[\begin{array}{l}
p_{1} \\
u_{1} \\
p_{2} \\
u_{2} \\
u_{3}
\end{array}\right]} \\
& =\overrightarrow{0} .
\end{aligned}
$$

The geometric setup of the model of Fig. 11 depends on the width $W_{3}$ and area $S_{3}$ of the inter-resonator section and width of the resonator section $W_{1}$. The influence of these parameters on the frequency response is investigated by introducing the non-dimensionalized width $\alpha_{1}=W_{1} / W$ and $\alpha_{3}$ $=W_{3} / W$, and non-dimensionalized effective area $\gamma=S_{3} / A$, where $A$ is the cross sectional area of the cavity. The model is assumed to be geometrically symmetric so that $W_{1}=W_{2}$, $V_{1}=V_{2}, S_{1}=S_{2}$ and $L_{1}^{\prime}=L_{2}^{\prime}=L^{\prime}$.

Solving the system of Eq. (17) for $f=\omega / 2 \pi$ yields an expression for the natural frequency:

$$
f_{2}=\frac{c}{2 \pi} \sqrt{\frac{S}{V L^{\prime}}+\frac{E}{W^{2}}}
$$

where

$$
E=\left(\frac{2 \gamma}{\alpha_{1} \alpha_{3}}\right) .
$$

Compared to the resonance frequency of a single Helmholtz resonator [Eq. (1)] there is an additional term depending on the spanwise length $W$.

The natural resonance frequency for a system of arbitrary number of resonators in the cavity span $n=1,2,3, \ldots$ can be found by organizing the the section width into a number of the dual resonator systems of Eq. (18) with reduced total span $W / n-1$. This will give:

$$
f_{n}=\frac{c}{2 \pi} \sqrt{\frac{S}{V L^{\prime}}+\frac{E(n-1)^{2}}{W^{2}}} .
$$

For $n=1$, Eq. (1) is retrieved.

Using spanwise integration of the ART results bandfiltered cavity volume shown in Fig. 9, the magnitudes of $\alpha_{1}$, $\alpha_{2}$ can be estimated. This will give $\alpha_{1} \approx / 1 \pi, \alpha_{3} \approx 2 / \pi$. Note that due to the gradual change in span, the widths of the inter-resonator and resonator sections do not add up to the total spanwise width exactly, but partly overlap. Filling in will give:

$$
f_{n}=\frac{c}{2 \pi} \sqrt{\frac{S}{V L^{\prime}}+\beta\left(\frac{\pi(n-1)}{W}\right)^{2}},
$$

with $\beta$ of order 1 , accounting for variations in resonator effective cross-section and widths. The used lumped mass model derivation indicates that $n$ interacting Helmholtz resonators are present in the span. It should be noted however that Eq. (21) can also be interpreted as combination of Helmholtz resonance and spanwise planar wave modes.
TABLE IV. Comparison analytical model multiple Helmholtz resonators with flow run higher mode frequencies, $\beta=0.97$. Large $(0.5 \mathrm{~m})$ and small $(0.3 \mathrm{~m})$ span flow experiments included.

\begin{tabular}{ccc}
\hline \hline $\begin{array}{c}\text { Large span }(0.5 \mathrm{~m}) \\
\text { no. of sections }\end{array}$ & $\begin{array}{c}\text { Exp. freq. } \\
(\mathrm{Hz})\end{array}$ & $\begin{array}{c}\text { flow-run based }) \\
(\mathrm{Hz})\end{array}$ \\
\hline 1 & $830(\mathrm{set})$ & 830 \\
2 & 897 & 900 \\
3 & 1074 & 1050 \\
4 & 1318 & 1300 \\
5 & 1597 & 1600 \\
6 & 1897 & 1900 \\
& & \\
Small span $(0.3 \mathrm{~m})$ & & 830 \\
no. of sections & & 1000 \\
1 & $830(\mathrm{set})$ & 1420 \\
\hline 3 & 1006 & \\
\hline
\end{tabular}

\section{B. Comparison of model with experiment}

The derived analytical model for multiple Helmholtz resonators of Eq. (21) is compared with the experimental flow run results in Table IV. When $\beta=0.97$ is chosen, a good match with the experimentally obtained modes can be found.

Table IV includes flow run data from the $0.3 \mathrm{~m}$ and 0.5 m span cavity flow run experiments. The base Helmholtz resonance effective length $L^{\prime}$ is set to match the base resonance frequency. The analytical resonance frequencies are all within a few percent of the observed experimental values. This result is obtained by using only one chosen variable $\beta$ and thus the analytical model seems to be able to describe the observed physical behavior.

\section{CONCLUSIONS}

In this paper the aeroacoustic response of slender covered cavities has been presented. In the investigated geometries several aeroacoustic resonances occur. The base resonance mechanism is of a simple slender Helmholtz type. The higher resonances show spanwise variations that do not correspond to pure simple standing wave modes. All observed resonances are excited by the first stage hydrodynamic mode.

The acoustic response (no flow) of the geometry is simulated using a Lattice Boltzmann method. This simulation is validated by comparing it with an experimental acoustic response test. The acoustic simulations revealed the mechanism for the higher resonance modes of the flow experiments by frequency band-filtering the simulation pressure signal in the entire cavity internal volume. The cavity higher modes with spanwise variation can be interpreted as spanwise sections that act as separate Helmholtz resonators interacting with each other.

A lumped mass analytical model for the coupled resonator sections is derived based on the experimental and numerical results that matches the observed mode frequencies well. The resonance frequency depends on the cavity total spanwise width. In the limit of one resonator, the original slender Helmholtz equation is retrieved. 


\section{ACKNOWLEDGMENTS}

We would like to thank Prof. Hirschberg from Eindhoven University of Technology for the helpful advice on cavity acoustics and advice on design of the experimental setup. We also would like to thank Stefan Bernardi at the Delft university of Technology low speed laboratory for helping setting up the wind tunnel experiments. From Exa Corp. we would like to thank Dr. Bernd Crouse, Dr. David Freed and Gana Balasubramanian for the support. At the aerodynamics department of Delft University we would like to thank Dr. Leo Veldhuis for his helpful discussions and Prof. Fulvio Scarano for his experimental expertise.

${ }^{1}$ J. E. Rossiter, Wind-Tunnel Experiments on the Flow Over Rectangular Cavities at Subsonic and Transonic Speeds, 1st ed. (HMSO, London, 1967), pp. 1-32.

${ }^{2}$ C. Noger, J. Patrat, J. Peube, and J. Peube, "Aeroacoustical study of the TGV pantograph recess," J. Sound Vib. 231, 563-575 (2000).

${ }^{3}$ P. Radavich, A. Selamet, and J. Novak, "A computational approach for flow-acoustic coupling in closed side branches," J. Acoust. Soc. Am. 109, 1343-1353 (2001).

${ }^{4}$ S. Dequand, S. Hulshoff, and A. Hirschberg, "Self-sustained oscillations in a closed side branch system," J. Sound Vib. 265, 359-386 (2003).

${ }^{5}$ S. Dequand, X. Luo, J. Willems, and A. Hirschberg, "Helmholtz-like resonator self-sustained oscillations, Part 1: Acoustical measurements and analytical models," AIAA J. 41, 408-415 (2003).

${ }^{6}$ S. Dequand S. Hulshoff, H. Van Kuijk, J. Willems, and A. Hirschberg, "Helmholtz-like resonator self-sustained oscillations, Part 2: Detailed flow measurements and numerical simulations," AIAA J. 41, 416-423 (2003).

${ }^{7}$ S. Dequand, J. F. H. Willems, M. Leroux, R. Vullings, M. van Weert, C. Thieulot, and A. Hirschberg, "Simplified models of flue instruments: Influence of mouth geometry on the sound source," J. Acoust. Soc. Am. 113, 1724-1735 (2003)

${ }^{8}$ J. Coltman, "Jet drive mechanism in edge tones and organ pipes," J. Acoust. Soc. Am. 60, 725-733 (1976).

${ }^{9}$ S. A. Elder, "On the mechanism of sound production in organ pipes," J. Acoust. Soc. Am. 54, 1554-1564 (1973).

${ }^{10}$ B. Crouse, S. Senthooran, G. Balasubramanian, D. Freed, S. Noelting, L. Mongeau, and J. Hong, "Sunroof buffeting of a simplified car model: Simulations of the acoustic and flow-induced responses," SAE Paper No. 2005-01-2498, 2005, pp. 2498-2513.

${ }^{11}$ D. Ricot, V. Maillard, and C. Bailly, "Numerical simulation of the unsteady flow past a cavity and application to sunroof buffeting," in the Seventh AIAA/CEAS Aeroacoustics Conference, Maastricht, The Nether- lands (2001), AIAA Paper No. 2001-2112, pp. 1-11.

${ }^{12} \mathrm{U}$. Ingard, "On the theory and design of acoustic resonators," J. Acoust. Soc. Am. 25, 1037-1061 (1953).

${ }^{13} \mathrm{M}$. Howe, "Low Strouhal number instabilities of flow over apertures and wall cavities," J. Acoust. Soc. Am. 102, 772-780 (1997).

${ }^{14}$ G. Kooijman, A. Hirschberg, and J. Golliard, "Acoustical response of orifices under grazing flow: Effect of boundary layer profile and edge geometry," J. Sound Vib. 315, 849-874 (2008).

${ }^{15}$ P. A. Nelson, N. A. Halliwell, and P. E. Doak, "Fluid dynamics of a flow excited resonance, Part I: Experiment," J. Sound Vib. 78, 15-38 (1981).

${ }^{16}$ P. A. Nelson, N. A. Halliwell, and P. E. Doak, "Fluid dynamics of a flow excited resonance, Part II: Flow acoustic interaction," J. Sound Vib. 91, 375-402 (1983).

${ }^{17}$ B. S. Henderson, "Category 6 automobile noise involving feedbackSound generated by low speed cavity tones," NASA Technical Report No. NASA/CP-2000-209790, 2000, pp. 95-100.

${ }^{18}$ B. S. Henderson, "Category 5: Sound generation in viscous problems, problem 2: Sound generation by flow over a cavity," NASA Technical Report No. NASA/CP-2004-212954, 2004, pp. 71-77.

${ }^{19}$ L. Mongeau, J. D. Bezemek, and R. Danforth, "Pressure fluctuations in a flow-excited door gap model," Paper No. SAE-971923, 1997, pp. 1-7.

${ }^{20}$ L. Larchevêque, P. Sagaut, T. H. Lê, and P. Comte, "Large-eddy simulation of a compressible flow in a three dimensional open cavity at high Reynolds number," J. Fluid Mech. 516, 265-301 (2004).

${ }^{21}$ S. Elder, T. Farabee, and F. DeMetz, "Mechanisms of flow-excited cavity tones at low Mach number," J. Acoust. Soc. Am. 72, 532-549 (1982).

${ }^{22}$ X. Gloerfelt, "Cavity noise," http://sin-web.paris.ensam.fr/squelettes/ref_ biblio/Gloerfelt_VKI_2009a.pdf (Last viewed 10/26/09).

${ }^{23}$ F. M. White, Viscous Fluid Flow, 2nd ed. (McGraw-Hill, New York, 1991), pp. 409 and 413.

${ }^{24}$ A. Hirschberg and S. W. Rienstra, "An introduction to aeroacoustics," http://www.win. tue.nl/ sjoerdr/papers/les-swr-mh.pdf (date last viewed 10/26/09) (2004).

${ }^{25}$ S. Succi, The Lattice Boltzmann Equation for Fluid Dynamics and Beyond (Oxford University Press, Oxford, 2001), pp. 11-13.

${ }^{26}$ B. Crouse, D. M. Freed, G. Balasubramanian, S. Senthooran, P. Lew, and L. Mongeau, "Fundamental aeroacoustic capabilities of the latticeBoltzmann method," AIAA Conference, Cambridge, MA (2006), AIAA Paper No. 2006-2571, pp. 2571-2588.

${ }^{27}$ A. Berlanger, M. Meskine, B. Caruelle, and K. Debatin, "Aero-acoustic simulation of a double diaphragm using lattice Boltzmann method," in the 11th AIAA/CEAS Aeroacoustics Conference, Monterey, CA (2005), AIAA Paper No. 2005-2917, pp. 2917-2929.

${ }^{28}$ A. R. da Silva and G. P. Scavone, "Lattice Boltzmann simulations of the acoustic radiation from waveguides," J. Phys. A 40, 397-408 (2007).

${ }^{29}$ X. M. Li, R. C. Leung, and R. M. C. So, "One-step aeroacoustics simulation using lattice Boltzmann method," AIAA J. 44, 78-89 (2006). 